\title{
Special Issue: Qualitative Marketing Forschung - Konzeptionelle Entwicklungen und Methodische Trends
}

\author{
Rudolf R. Sinkovics
}

Online veröffentlicht: 31. März 2009

(C) Springer-Verlag 2009

\section{Über den Special Issue}

Qualitative Forschungsmethoden haben in den letzten Jahren kontinuierlich an Bedeutung gewonnen. Offene und interpretative Ansätze zählen mittlerweile zum Standardwissen der Methodentoolbox, welche DoktoratsstudentInnen in der KonsumentInnenforschung, im strategischen Marketing oder auch im International Business kennenlernen. Neuere Buchpublikationen (siehe etwa Buber u. Holzmüller 2007; Marschan-Piekkari u. Welch 2004) und Special Issues und Leitartikel führender wissenschaftlicher Publikationen wie etwa International Marketing Review (IMR), International Business Review (IBR), Academy of Management Journal (AMJ), oder Management International Review (MIR) unterstreichen die Bedeutung die diesem Themenfeld zukommt (Piekkari u. Welch 2006; Rynes u. Gephart Jr. 2004; Sinkovics u. Salzberger 2006; Suddaby 2006). Gleichermaßen haben technologische und software-basierte Fortschritte die Möglichkeiten und das Spektrum qualitativer Methoden revolutioniert und es sind in dieser Hinsicht in der Zukunft noch viele Initiativen zu erwarten.

Andererseits dominieren quantitative Studien weiterhin die akademischen Publikationslandschaft (siehe etwa Yang et al. 2006) und das Wissen über qualitative Forschungsmethoden findet nicht notwendigerweise Anwendung. Ein Grund dafür liegt zweifellos in der Publikationspolitik von Journalen und der herrschenden Forschungsparadigmatik (Jack u. Westwood 2006). Auch wird vielfach die unklare, inhärent subjektive und nicht genau formalisierte Doku-

R. R. Sinkovics (

Manchester Business School, The University of Manchester,

Booth Street West, M15 6PB Manchester, UK

E-Mail: Rudolf.Sinkovics@manchester.ac.uk mentation qualitativer Forschung für die Zurückhaltung verantwortlich gemacht, mit der man bezüglich Nutzung und Veröffentlichung qualitativer Ergebnisse konfrontiert ist (Rynes u. Gephart Jr. 2004; Sinkovics et al. 2005). „There are probably rules for writing the persuasive, memorable and publishable qualitative research article but, rest assured, no one knows what they are" (Van Maanen 1998).

Zielsetzung dieses Special Issues ist es daher, konzeptionelle und methodische Forschungsarbeiten qualitativer Natur mit besonderem Bezug zum Funktionalbereich Marketing zu betrachten. Es geht darum Innovationen qualitativer Forschung vorzustellen und konstruktive Anwendungsmöglichkeiten für diese Forschungsmethoden darzulegen. Durch die Offenlegung der Forschungspragmatik und der Dokumentation spezifischer Routinen die qualitativen Studien zugrunde liegen, kann ein Beitrag für die Erhöhung der Transparenz und der Glaubwürdigkeit qualitativer Methoden geliefert werden (Sinkovics et al. 2008). Dies soll mithelfen zukünftige Marketingforschungsprojekte $\mathrm{zu}$ unterstützen, methodisch durchsetzungskräftiger zu machen und Zukunftsentwicklungen für den Gegenstandsbereich zu illustrieren.

Insgesamt wurden 11 Manuskripte für diesen Special Issue eingereicht. Aus diesen Einreichungen wurden insgesamt sechs Manuskripte in einem double-blind Review-Prozess zur Reife gebracht. Die Zahl der eingereichten Manuskripte ist angesichts des steigenden Publikationsdrucks und der damit einhergehenden Wichtigkeit von publizierten Forschungsnachweisen überraschend gering. Dies dürfte jedoch zum einen mit der Ausrichtung des Special Issues zu erklären sein, zum anderen mit der im deutschsprachigen Wissenschaftswelt zunehmenden Anglikanisierung der Publikationen (Sinkovics u. Schlegelmilch 2000). Die deutsche Sprache als Trägermedium für Diskussionen konzeptioneller und empirischer Forschung 
steht zusehends am Rande der Wahrnehmung. Damit einher geht die Marginalisierung deutschsprachiger Journale und auch die Zeitschrift der markt ist mit diesem Phänomen konfrontiert. Vor dem Hintergrund dieser komplexen Marktsituation, die ich persönlich nicht einfach mit der österreichischen Redensart „so ist das nun einmal” abtun möchte, freut es mich besonders dass in diesem Special Issue eine ansatzweise Entschleunigung und Umkehrung dieses Trends zu verzeichnen ist. Neben deutschsprachigen Beiträgen ist es gelungen englischsprachige Manuskripte zu rekrutieren und wir haben AutorInnen aus den USA, Spanien, Taiwan und England in diesem Special Issue.

\section{Manuskripte in diesem Special Issue}

Die Manuskripte des Special Issues sind inhaltlich geordnet. Zunächst werden zwei konzeptionelle Beiträge vorgestellt, welche sich dem Thema qualitativer Marketingforschung unter konzeptionellen Gesichtspunkten und die einschlägige Literatur nähern. In weiterer Folge werden eine Reihe von Beiträgen geliefert, die methodisch und empirische Befunde liefern.

Im erstes Beitrag mit dem Titel „Qualitative und kombinierte Methoden in der wissenschaftlichen Marketingforschung: Theoretische Betrachtung \& Literaturanalyse" untersucht Katharina J. Auer-Srnka den Bedarf an alternativen Forschungszugängen sowie des Potenzials qualitativer Methoden. Sie untersucht im Rahmen einer Literaturanalyse welche qualitativen Beiträge in den elf höchstgereihten wissenschaftlichen Zeitschriften im Bereich Marketing zum Einsatz kommen. Ihre Untersuchung liefert ein Verständnis über Qualitätskriterien und Gütemaße in den untersuchten qualitativen Studien und bietet Ansatzpunkte für den Einsatz sowie eine bessere Publizierbarkeit qualitativer bzw. kombinierter Forschung.

Ruey-Jer 'Bryan' Jean, Chia-Ling 'Eunice' Liu und Claudia Klausegger widmen sich dem Thema qualitativer Methoden am Beispiel der 'emerging markets' im asiatischpazifischen Raum. Sie liefern einen Literaturüberblick empirischer in den vier führenden Zeitschriften zum International Business (IB) und präsentieren anschließend eine Fallstudie, die auf Basis interviewgestützter qualitativer Forschung in taiwanesischen Elektronikunternehmen entwickelt wurde. Ihr Forschungsprojekt liefert ein Beispiel wie qualitative Forschung die Gültigkeit von Forschungsergebnissen verbessern kann und neues Wissen in Bezug auf die Wachstumsmärkte Asiens generieren kann.

Der methodische Teil der Arbeiten in diesem Special Issue wird durch Pervez N. Ghauri und Rebecca Firth eingeleitet. Ihr Manuskript „Die Formalisierung von Fallstudienforschung im Internationalen Business" versucht
Qualitätsprobleme bei der Durchführung und Dokumentation von Fallstudien-basierter Forschung zu bewältigen. Vor dem Hintergrund eines umfangreichen Fallstudienprojektes im Service-sektor das sich mit „Linkages” zwischen UKbasierten multinationalen Unternehmen und lokalen Firmen in China und Korea auseinandersetzt, wird die Nützlichkeit von computerbasierter Analyse für die Systematisierung und Qualitätsverbesserung von qualitativer Forschung dargestellt.

Ebru Ulusoy und A. Fuat Firat greifen mit ihrem Artikel "Incorporating the visual into qualitative research: Living a theme as an illustrative example" einen Trend in der qualitativen Marketingforschung auf und illustrieren dies in ihrem Videographie-Ansatz. Ihre videographische Studie illustriert wie visuelle Elemente in Forschungsprojekte inkorporiert werden können und sie bieten dies als Zusatz zu normalerweise gebräuchlichen Wortbausteinen an. Videos sind beachtenswerte Hilfsmittel für die Repräsentation des Forschungskontextes oder die Entwicklung von Forschungsfragen.

„Die Entstehung von Vertrauen im persönlichen Verkauf - Eine qualitative Analyse von Gesprächseröffnungen", so der Titel des Beitrages von Renate Buber und Horst Reiger. Die Autoren skizzieren den Forschungsstand zum Persönlichen Verkauf und zur Bedeutung von Vertrauen im Marketing und untersuchen anschließend wie mittels ritueller Interaktionsformen im konkreten Verkaufsgespräch im B-to-C Bereich Ver- bzw. Misstrauen aufgebaut wird. Dazu analysieren sie sprachliche dialogische Äußerungen in Eröffnungssequenzen in face-to-face Verkaufssituationen am Beispiel von Baumärkten und Modeboutiquen auf ihre vertrauensbildende latente Struktur. Unter methodologischen Gesichtspunkten zeigen die Autoren die Einsatzmöglichkeit hermeneutischer Textinterpretation für die Analyse von Gesprächsritualen.

Der letzte Beitrag stammt von Rudolf R. Sinkovics, Elfriede Penz und Francisco Jose Molina Castillo. Unter dem Titel „Qualitative Analyse von Online Communities für Neuproduktentscheidungen" nähert sich dieser Beitrag dem Thema neuer Kommunikationsformen an und prüft wie diese für Produktentscheidungen von Firmen verwendet werden können. Online Communities werden dabei als eine kostengünstige und vielfältige Alternative für qualitative Marktforschung vorgestellt. Unter Nutzung der qualitativen Analysesoftware Nvivo werden Möglichkeiten vorgestellt, wie qualitative Information aus Online Communities für Analysen verfügbar gemacht werden können und wie diese als Grundlage für Neuprodukt- und Produktvariationsentscheidungen Verwendung finden können.

Danksagung Besonderer Dank gilt den Herausgebern der Zeitschrift der markt, Thomas Salzberger und Arne Floh, die mich zu diesem Special Issue angeregt haben. Darüberhinaus bin ich jenen Kollegen 
und Kolleginnen zu Dank verpflichtet, die durch ihre Zeitinvestitionen und Bemühungen der Qualitätssicherung im Review-Prozess wichtige Unterstützung vollbracht haben. Diese uneigennützige Unterstützung war für die qualitative Weiterentwicklung der Manuskripte von besonderer Bedeutung und hat für die Struktur und die Zusammengehörigkeit der Beiträge beigetragen. Die Review-KollegInnen sind nachfolgend in alphabetischer Reihenfolge gelistet:

Arne Floh, Wirtschaftsuniversität Wien, Austria

Thorsten Gruber, Manchester Business School, UK

Bianca Gusenbauer, Wirtschaftsuniversität Wien, Austria

Parissa Haghirian, Sophia University, Japan

Eva Hofmann, Wirtschaftsuniversität Wien, Austria

Erik Hölzl, Universität Wien, Austria

Hartmut Holzmüller, Universität Dortmund, Germany

Matthias Hossinger, Manchester Business School, UK

Katja Meier-Pesti, Psychonomics, Austria

Noemi Pezderka, Manchester Business School, UK

Christina Raftopoulou, Keele University, UK

Christoph Teller, University of Stirling, UK

Torsten Tomczak, Universität St. Gallen, Switzerland

Mo Yamin, Manchester Business School, UK

Anna Zueva, University of Bradford, UK

\section{Literatur}

Buber R, Holzmüller HH (2007) Qualitative Marktforschung: Konzepte - Methoden - Analysen. Gabler Verlag, Wiebaden
Jack G, Westwood R (2006) Postcolonialism and the politics of qualitative research in international business. Manag Int Rev 46(4):481-500

Marschan-Piekkari R, Welch C (eds) (2004) Handbook of qualitative research methods for international business. Edward Elgar, Cheltenham, UK

Piekkari R, Welch C (2006) Guest editors' introduction to the focused issue: qualitative research methods in international business. Manag Int Rev 46(4):391-396

Rynes S, Gephart Jr RP (2004) Qualitative research and the academy of management journal. Acad Manag J 47(4):454-462

Sinkovics RR, Penz E, Ghauri PN (2005) Analysing textual data in international marketing research. Qual Market Res: Int J 8(1):9-38

Sinkovics RR, Penz E, Ghauri PN (2008) Enhancing the trustworthiness of qualitative research in international business. Manag Int Rev 48(6):689-714

Sinkovics RR, Salzberger T (2006) Introduction to the special issue on ,issues and advances in international marketing research”. Int Market Rev 23(4):349-352

Sinkovics RR, Schlegelmilch BB (2000) Marketing academics in Austria, Germany and Switzerland: Humboldt's ideals give way to performance pressure. J Market Manag 16(6):745-759

Suddaby R (2006) From the editors: what grounded theory is not. Acad Manag J 49(4):633-642

Van Maanen J (1998) Different strokes: qualitative research in the administrative science quarterly from 1956 to 1996. In Van Maanen J (ed) Qualitative studies of organizations. Sage Publications, Thousand Oaks

Yang Z, Wang X, Su C (2006) A Review of research methodologies in international business. Int Busin Rev 15(6):601-617 